\title{
Effect of Co Doping on Structural and Optical Properties of Zinc Oxide Nanoparticles Synthesized by Sol-Gel Method
}

\author{
Aravapalli Vanaja1, Karumuri Srinivisa Rao' \\ ${ }^{1}$ Department of Physics, Lingaya's University, Old Faridabad, India \\ ${ }^{2}$ Department of ECE, KL University, Andhra Pradesh, India \\ Email: vanajatunuguntla@yahoo.com
}

Received 27 July 2015; accepted 13 February 2016; published 16 February 2016

Copyright (C) 2016 by authors and Scientific Research Publishing Inc.

This work is licensed under the Creative Commons Attribution International License (CC BY). http://creativecommons.org/licenses/by/4.0/

(c) (i) Open Access

\begin{abstract}
In the present work Pure and Cobalt (Co) doped Zinc Oxide (ZnO) Nanoparticles were synthesized by Sol-gel method. The effect of Co doping on structural and optical properties of Nanoparticles were investigated using X-Ray Diffraction (XRD), Scanning Electron Microscopy (SEM) and Fourier Transform Infrared (FTIR) spectroscopic characterization techniques. The XRD spectrum revealed the formation of Wurtzite structure of ZnO. The structural properties of nanoparticles including particle size and lattice strain were calculated from XRD data. SEM shows the morphology of Nanoparticles. The presence of functional groups was analyzed from FTIR spectra. The properties of Cobalt doped zinc oxide nanoparticles prepared through simple cost effective, Sol-gel method can be used in optoelectronics and thermally stable devices.
\end{abstract}

\section{Keywords}

Nanoparticles, Structural Properties, Optical Properties, X-Ray Diffraction, Scanning Electron Microscopy, ZnO

\section{Introduction}

In the recent years, researchers have been looking intensively at the use of nanomaterials due to their outstanding chemical and physical properties compared to bulk materials. Nanoparticles can contribute to stronger, lighter, cleaner and smarter surfaces and systems [1]. Today nano-structured materials have already been used in the manufacture of scratch proof eyeglasses, crack-resistant paints and anti-graffiti coatings for walls, transparent sunscreens, stain-repellent fabrics, self-cleaning windows and ceramic coatings for solar cells [2]. 
The development of innovative materials is the essential part of research [3]. Zinc oxide is an inorganic compound which has its own importance due to wide range of applications e.g., gas sensor, chemical sensor, biosensor, cosmetics, storage, optical and electrical devices, window materials for displays, solar cells, and drugdelivery [4]. $\mathrm{ZnO}$ is an attractive material for short-wavelength optoelectronic applications owing to its wide band gap $3.37 \mathrm{eV}$, large bond strength, and large exciton binding energy $(60 \mathrm{meV})$ at room temperature and the missing absorbance of visible light makes this material one of the best transition metal oxide nanoparicles so far [5]. By using $\mathrm{ZnO}$ nanoparticles, it is possible to tune the optical and electrical properties in a wide range [6]. As a wide band gap material, $\mathrm{ZnO}$ is used in solid state blue to ultraviolet (UV) opto-electronics, including laser developments [7]. In addition, due to its non-centrosymmetric crystallographic phase, $\mathrm{ZnO}$ shows the piezoelectric property, which is highly useful for the fabrication of devices such as electromagnetic coupled sensors and actuators [8].

The structural, morphological and optical properties of $\mathrm{ZnO}$ nanoparticles are investigated regarding doping [9]. The intensity behind doping is to modify the properties of nanoparticles. Further, to enhance the magnetization and their utilization in DMS and spintronics, doping of transition metal ions such as $\mathrm{Ni}, \mathrm{Co}, \mathrm{Mn}$, and $\mathrm{Ni}$ are carried out by the researchers [10]. A number of studies have reported on Co doped $\mathrm{ZnO}$ nanoparticles [11]. Talaat 2013 synthesized Co doped $\mathrm{ZnO}$ nanoparticles with an average size of 5 - $11 \mathrm{~nm}$ using Sol gel method. H. J. Sugahara in his studies reported an increase in photo luminescence properties by Co doping. Shalendra Kumar reported room temperature ferromagnetism of Co doped $\mathrm{ZnO}$. Talaat $\mathrm{M}$. reported the increase in the band gap by Co doping. From theoretical and experimental point of view the transition metal ions doped- $\mathrm{ZnO}$ nanoparticles still continue to be an open topic [12].

In this work we report an investigation on structural and optical properties of $\mathrm{ZnO}$ particles doped with Cobalt ions [13]. The advantages of using sol-gel processing instead of high temperature processing methods are: low synthesis temperature, high purity, novel materials, and low capital costs [14]. The effect of cobalt incorporation on structural and optical properties was investigated using X-ray diffraction (XRD), Scanning electron microscopy (SEM) and Fourier transform infrared spectroscopy (FTIR) techniques [15].

\section{Experimental Procedure}

\subsection{Chemicals Required}

Zinc Acetate $\left(\mathrm{ZnCl}_{2}\right)$, Potassium Hydroxide $(\mathrm{KOH})$, Cobalt Acetate $\left(\mathrm{Co}\left(\mathrm{C}_{2} \mathrm{H}_{3} \mathrm{O}_{2}\right)_{2}\right)$ and Ethanol $\left(\mathrm{C}_{2} \mathrm{H}_{6} \mathrm{O}\right)$ were purchased and used without any purification. The chemicals used are of analytical grade purity.

\subsection{Apparatus Required}

Magnetic stirrer (REMI, 1, MLH), Glass jars, Centrifuge Mission (REMI), Micro oven (VSE 230 A/C) and High Precision Balance (INFRA).

\subsection{Procedure for Synthesis of Pure and Cobalt Doped ZnO Nanoparticles}

To synthesize Pure ZnO Nanoparticles, Aqueous Ethanol solution of $0.5 \mathrm{M}$ Zinc Acetate was kept under constant stiring using magnetic stirrer to dissolve completely Zinc Acetate for one hour and $0.5 \mathrm{M}$ aqueous ethanol solution of Potassium Hydroxide 0.5 M was also prepared in the same way with stirring of one hour. After complete dissolution of Zinc acetate, aqueous ethanol solution of potassium hydroxide was added under high speed constant stirring drop by drop (slowly for $45 \mathrm{~min}$ ) touching the walls of the vessel. The reaction was allowed to continue for 2 hours after the completion of reaction the solution sealed and settled over night. Further the supernatant solution separated carefully. The remaining solution centrifuged and precipitate removed. Thus, precipitated $\mathrm{ZnO}$ NPs cleaned three times with demonized water and ethanol to remove the byproducts which were bound with the nanoparticles. The solution then dried in an oven at about $60^{\circ} \mathrm{C}$. After drying $\mathrm{Zn}(\mathrm{OH})_{2}$ is completely converted to into $\mathrm{ZnO}[16]$.

For the synthesis of Co-doped $\mathrm{ZnO}$ nanopowder $0.5 \mathrm{M}$ concentration of cobalt acetate was added into the zinc solution before potassium hydroxide $\mathrm{KOH}$ solution and the same process repeated to obtain the Co doped $\mathrm{ZnO}$ nanoparticles. The samples were further characterized to analyze the effect of cobalt doping on properties nanoparicles. 


\section{Characterization}

\subsection{X-Ray Diffraction (XRD)}

$\mathrm{X}$-ray diffraction is now a common technique for the study of crystal structures and atomic spacing. The patterns for the $\mathrm{ZnO}$ nanoparticles were recorded using an X-ray diffractometer (PANLYTICAL) using secondary monochromatic $\mathrm{CuK}_{\alpha}$ radiation of wavelength $\lambda=0.1541 \mathrm{~nm}$ at $40 \mathrm{Kv} / 50 \mathrm{~mA}$ in the scan range $2 \theta=20^{\circ}$ to $90^{\circ}$. Samples were supported on a glass slide.

\subsection{Scanning Electron Microscopy (SEM)}

Morphology of the samples was investigated using scanning electron microscope (SEM with EDXA, Sirion) which also has been used for compositional analysis of the prepared $\mathrm{ZnO}$ nanoparticles. A drop of nanoparticles dissolved in methanol was placed on a copper grid.

\subsection{Fourier Transform Infrared (FTIR) Spectroscopy}

The optical characterization of nanopowder is performed using Fourier Transform Infrared spectroscopy (FTIR). The presence of certain functional groups in a molecule can be obtained from FTIR spectra.

\section{Results and Discussions}

\subsection{X-Ray Diffraction (XRD)}

Figure 1 and Figure 2 represents XRD pattern of pure and Co doped ZnO Nanopowders. A definite line broadening of the XRD peaks indicate the synthesized material consists of particles in nanoscale range. The Diffraction analysis provides detailed information on the structural properties like peak intensity, peak position and full width half maximum (FWHM) data. XRD pattern of Pure ZnO nanoparticles consist of diffraction peaks for $2 \theta$ $=31.92^{\circ}, 34.54^{\circ}, 36.398^{\circ}, 47.59^{\circ}, 56.59^{\circ}$ and $68.87^{\circ}$ respectively. XRD pattern of Co doped $\mathrm{ZnO}$ Nanoparticles consists of diffraction peaks for $2 \theta=18.44^{\circ}, 19.15^{\circ}, 23.73^{\circ}, 23.723^{\circ}, 24.07^{\circ}, 25.19^{\circ}, 27.09^{\circ}, 28.7^{\circ}, 29.52^{\circ}$, $32.526^{\circ}, 33.04^{\circ}, 33.945^{\circ}, 34.62^{\circ}, 38.45^{\circ}, 41.31^{\circ}, 41.942^{\circ}, 43.76^{\circ}, 47.76^{\circ}, 48.49^{\circ}, 49.78^{\circ}, 58.34^{\circ}, 68.00^{\circ}$ and $69.47^{\circ}$ respectively. The peaks clearly indexed the formation of Hexagonal wurtzite structure from JCPDS powder diffraction card file [17].

The crystallite size D is calculated using Scherer's formula

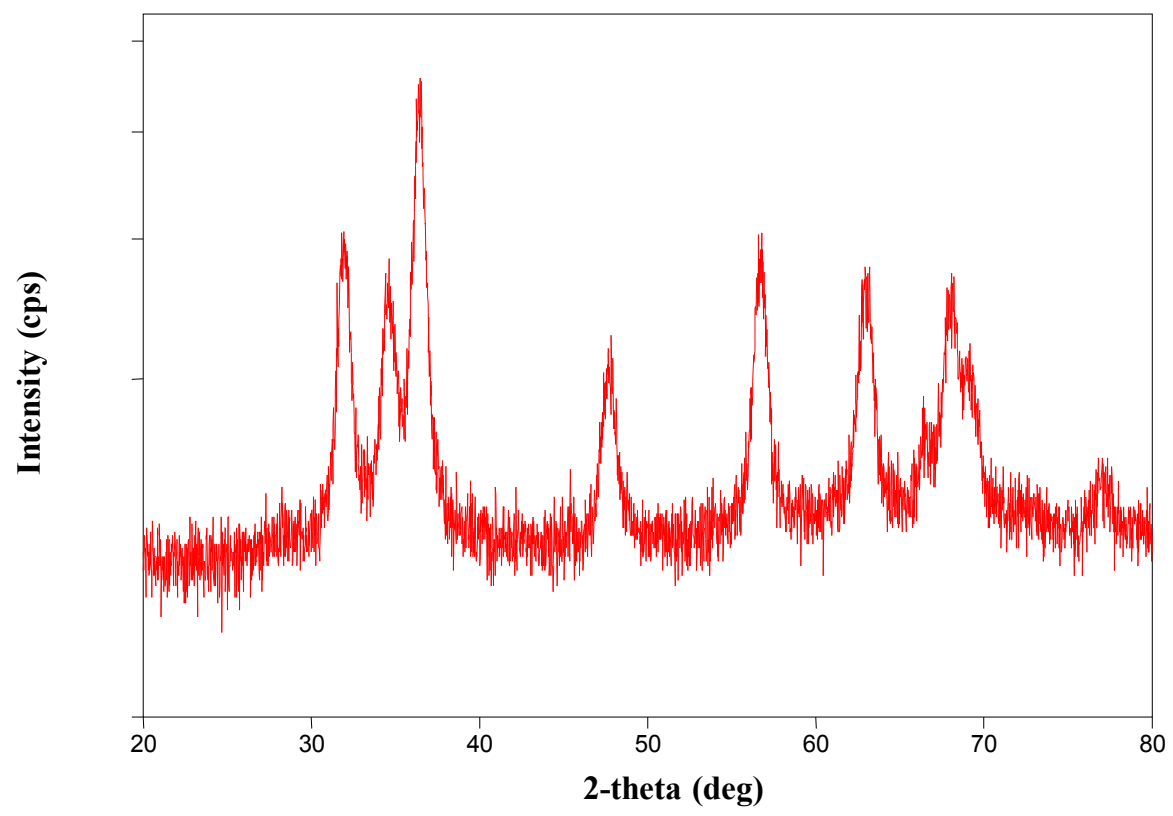

Figure 1. XRD spectrum of pure $\mathrm{ZnO}$ nanoparticles. 


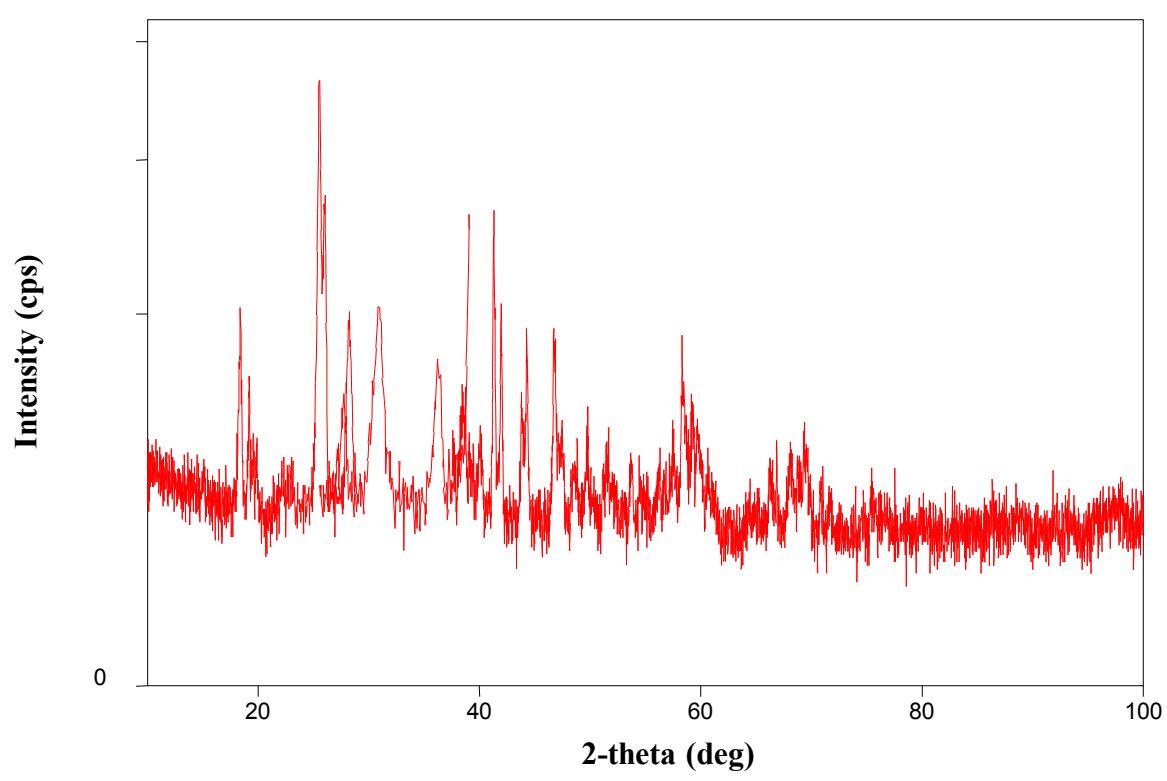

Figure 2. XRD spectrum of cobalt doped $\mathrm{ZnO}$ nanoparticles.

$$
D=k \lambda / \beta \cos \theta
$$

The Lattice strain induced in particles calculated using formula

$$
\varepsilon=\beta_{h k 1} / 4 \operatorname{Tan} \theta
$$

where $\mathrm{D}$ is the Average crystalline size, $\lambda$ represents wavelength of incident beam (1.5406 $\AA$ ), $\beta$ is the FWHM (Full Width Half Maxima) of the diffraction peak corresponding to $<101>$, $\mathrm{k}$ is the shape factor (it was set to 0.9 in our work), $\varepsilon$ is the lattice strain induced in nanoparticles and $\theta$ represents scattering angle of reflection.

The average crystalline size calculated from full width half maximum of high intense peak $<101>$ is found to be $10.34 \mathrm{~nm}$ for pure $\mathrm{ZnO}$ nanoparticles and $23.58 \mathrm{~nm}$ for Co doped $\mathrm{ZnO}$ Particles and corresponding lattice strain in the nanoparticles is found to be 0.0221 in the case of undoped $\mathrm{ZnO}$ and 0.0071 in the case of doped $\mathrm{ZnO}$ Nanoparticles. From spectrum it was clear that significant increase in the crystalline size is observed by doping with cobalt. This show snanoparticles possess good crystallinity. The reduction in the lattice strain explains the grain can grew much easier due to the incorporation of Co dopant.

\subsection{Scanning Electron Microscopy (SEM)}

SEM has opened doors in fields ranging from chemistry to engineering, allowing scientists to access new, useful information about microscopic processes with macroscopic implications. Figure 3 and Figure 4 represent SEM micrographs of Pure and Cobalt doped $\mathrm{ZnO}$ Nanoparticles observed at different magnifications. From micrographs, it is clear that the cauliflower like $\mathrm{ZnO}$ comprising of nanoclusters observed on the surface of the $\mathrm{ZnO}$ Nanoparticles and almost spherical shape morphology in doped ZnO. The morphological observation of SEM results indicates particles with less aggregation can be obtained from this method [18].

\subsection{Fourier Transform Infrared Spectroscopy (FTIR)}

Infrared spectroscopy is an important technique in organic chemistry. FTIR is one of the most widely used tools for the detection of functional groups in pure compounds and mixtures for compound comparison Formation of pure and doped $\mathrm{ZnO}$. Thespectra was recorded in the range $1000-3500 \mathrm{~cm}^{-1}$ as shown in Figure 5 and Figure 6. From spectra one can observe the shift in the vibration frequencies of $\mathrm{ZnO}$ Nanoparticles corresponding to $3443.46 \mathrm{~cm}^{-1}, 1632.66 \mathrm{~cm}^{-1}, 1362.17 \mathrm{~cm}^{-1}$ due to the incorporation of Cobalt ions in to the $\mathrm{ZnO}$ Wurtzite structure. The spectrum shows broad absorption peak between $3600-3200 \mathrm{Cm}^{-1}$ due to the $\mathrm{O}-\mathrm{H}$ stretching vibration of Water molecule. The absorption peak around $1632 \mathrm{~cm}^{-1}$ corresponds to $\mathrm{C}-\mathrm{H}$ stretching vibration and 

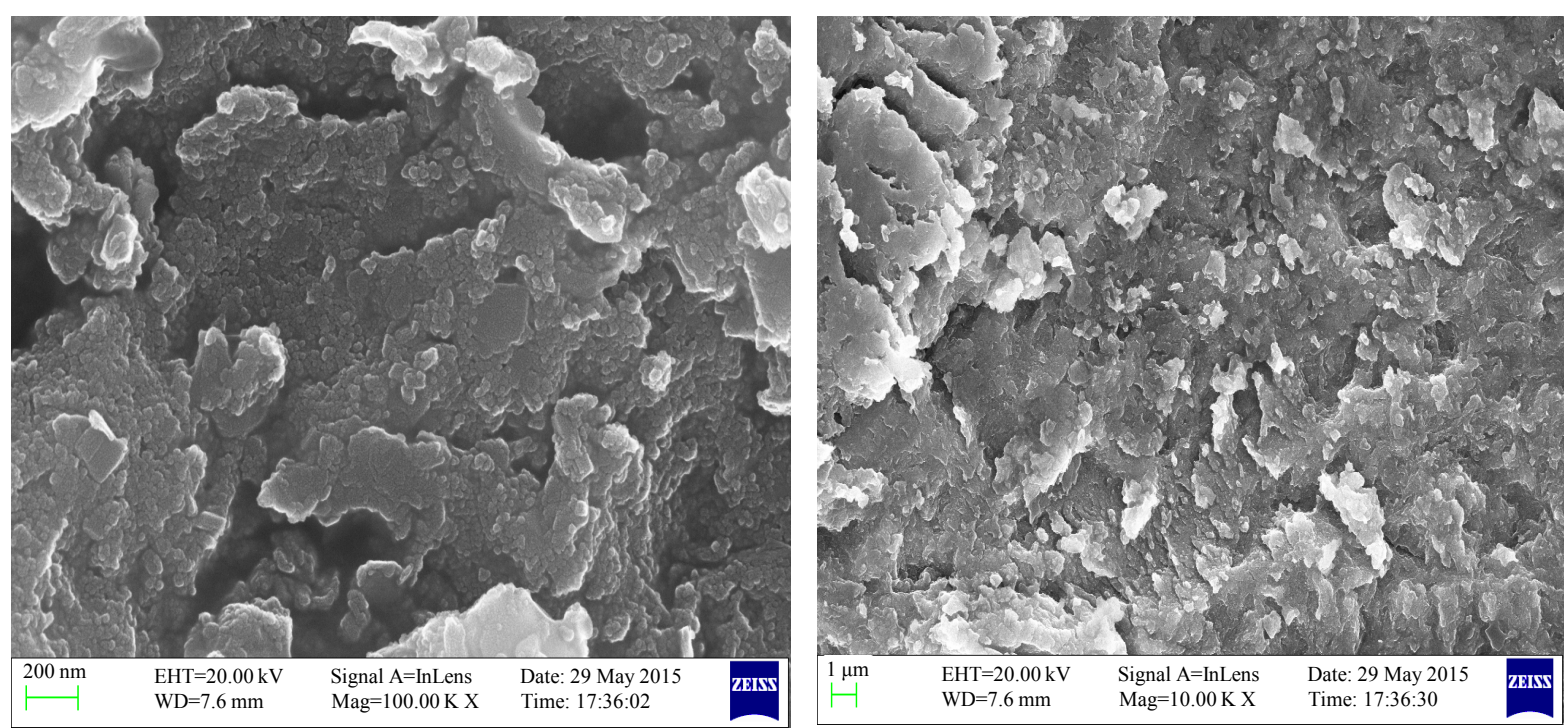

Figure3. SEM Micrographs Pure ZnO Nanoparticles.
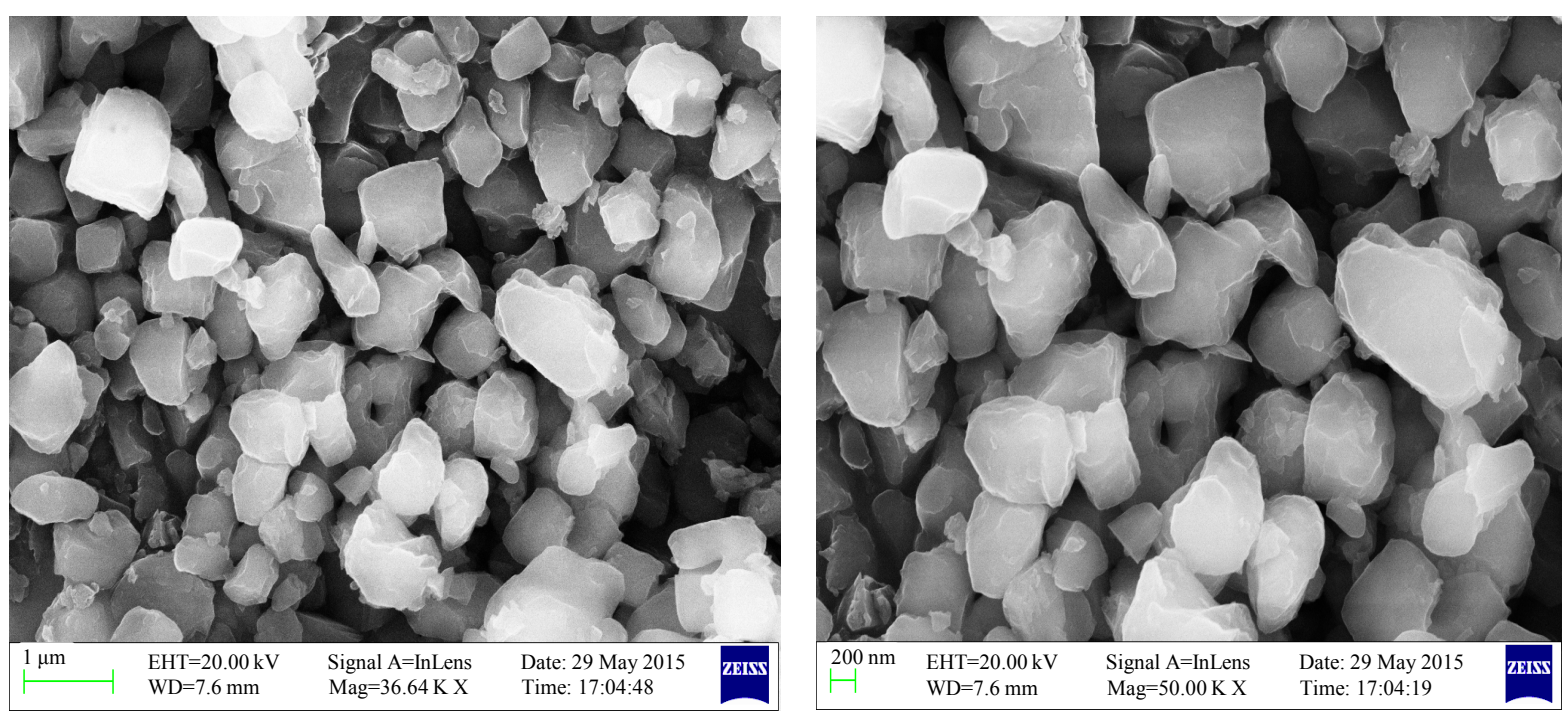

Figure 4. SEM Micrographs Co Doped ZnO Nanoparticles.

around $1300 \mathrm{~cm}^{-1}$ corresponds to $\mathrm{C}-\mathrm{O}$ symmetric stretching vibration. From spectra the main absorption between $600 \mathrm{~cm}^{-1}-1000 \mathrm{~cm}^{-1}$ corresponding to the stretching mode of $\mathrm{Zn}-\mathrm{O}$ in pure $\mathrm{ZnO}$ and characteristic absorption band due to the influence of Co is observed between $600-1000^{-1}$ in the spectra of doped ZnO Nanoparticles. These results further confirm the formation of Wurtzite structure in nanoparticles synthesized at room temperature.

\section{Conclusion}

In the present study Pure and Cobalt doped $\mathrm{ZnO}$ nanoparticles were synthesized via Sol Gel route and further analyzed through X-ray diffraction, scanning electron microscopy, Fourier transform infrared spectroscopyto analyzing the influence of $\mathrm{Co}$ doping on structural and optical properties of $\mathrm{ZnO}$ Nanoparticles. The XRD spectrum demonstrates that nanoparticles have the hexagonal wurtzite structure. The crystalline sizes of 10.34 $\mathrm{nm}$ and $23.58 \mathrm{~nm}$ are obtained from pure and Co doped ZnO. SEM images showed the formation of Cauli flower like $\mathrm{ZnO}$ composed of nanoclusters in the case of pure $\mathrm{ZnO}$ and almost spherical images due to the influence of Co doping. The vibration frequencies in the FTIR spectra further confirm the formation of wurtzite 


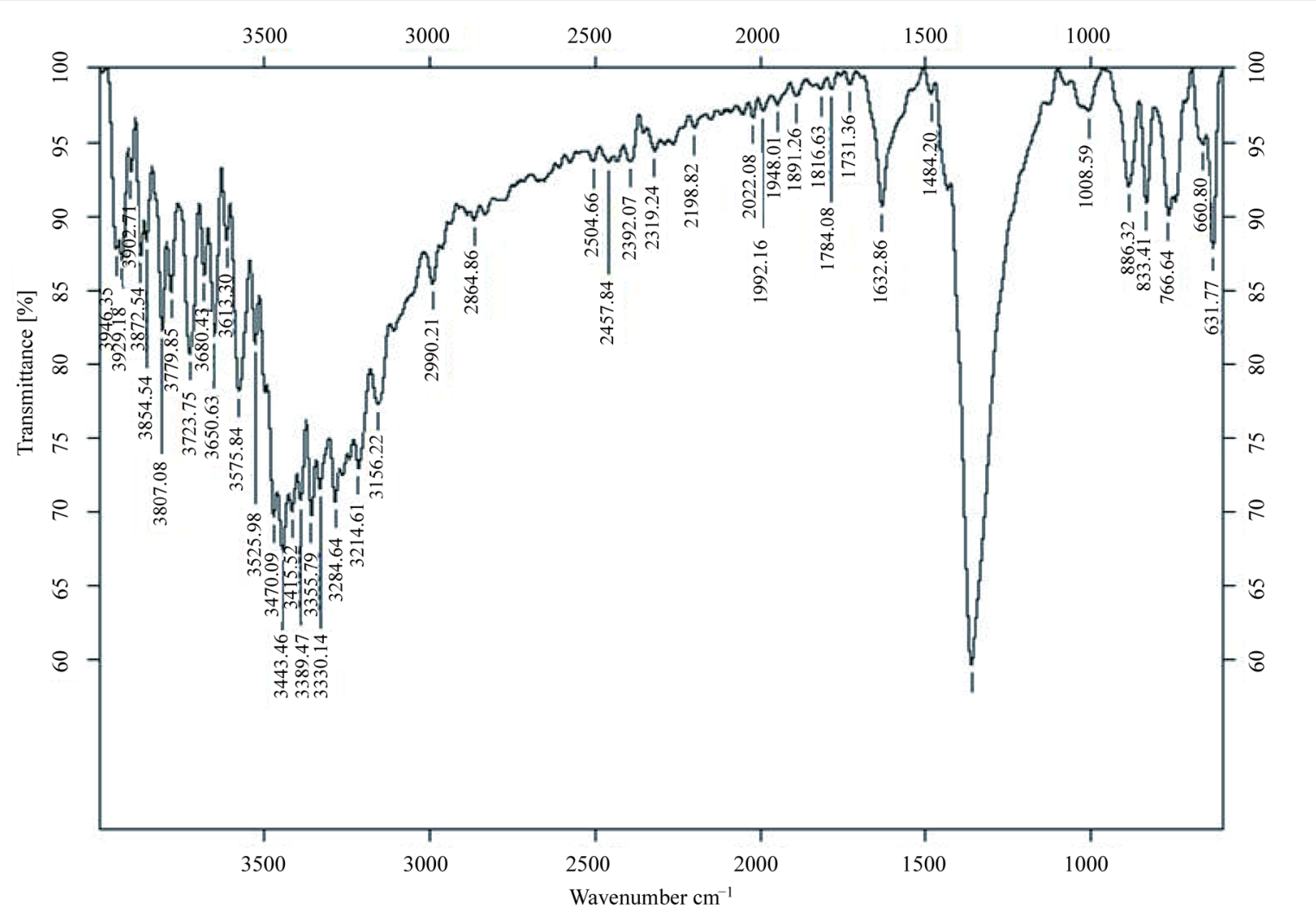

Figure 5. FTIR Spectrum of Pure ZnO Nanoparticles.

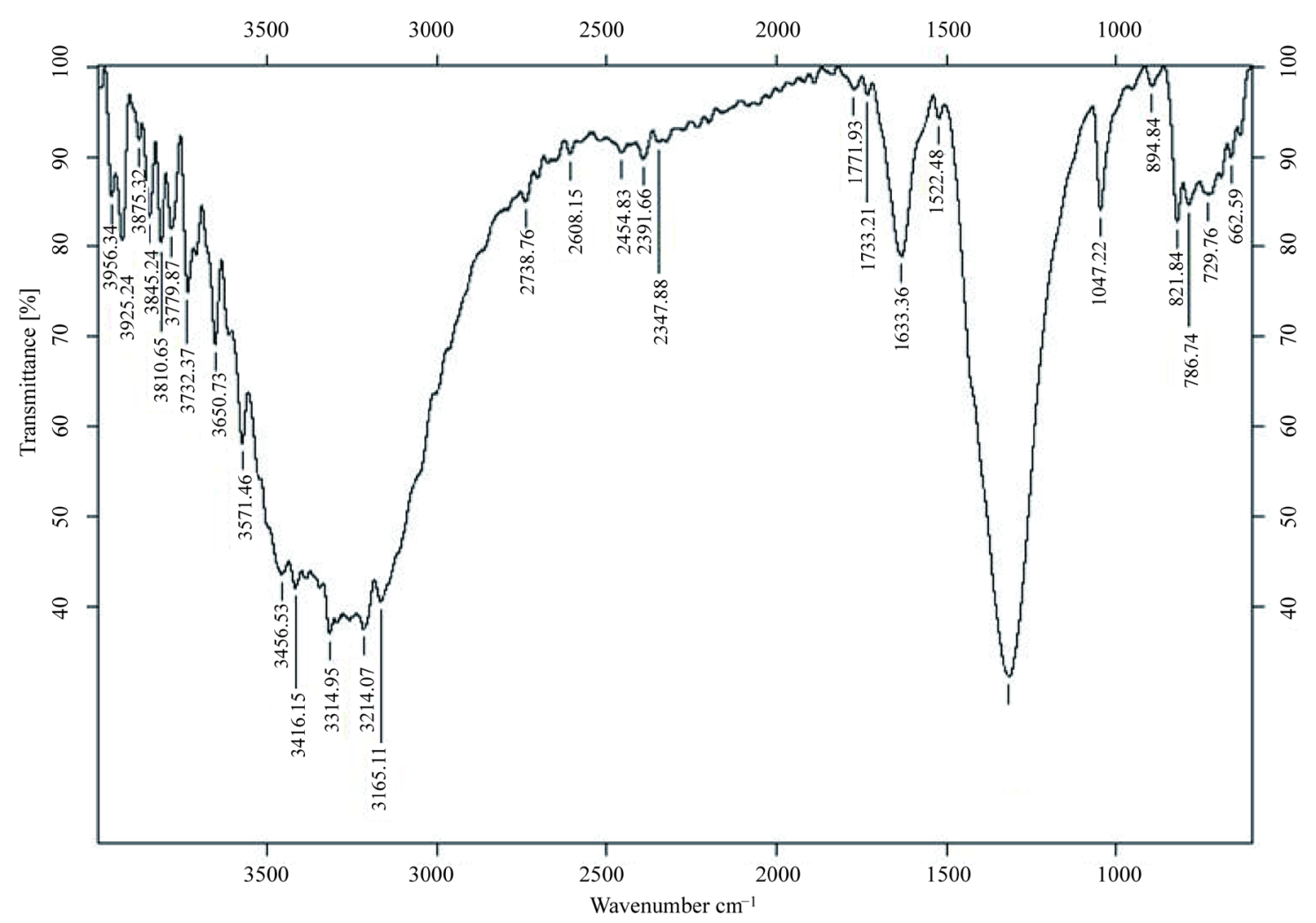

Figure6. FTIR Spectrum of Co Doped ZnO Nanoparticles. 
structure in $\mathrm{ZnO}$. Thus, the current doping method can be considered as another effective technique to modulate the properties of $\mathrm{ZnO}$ nanoparticles. The Co doped $\mathrm{ZnO}$ Nanoparticles synthesized by simple cost. Solgel route can be considered as an effective method to modify the properties of $\mathrm{ZnO}$ nanoparticles. The particles can be further utililized in the optoelcetonics and thermally stable devices.

\section{Acknowledgements}

The authors would like to thank SAIF IIT Chennai and Center for Nanoscience and Technology, Satyabhama University Chennai for proving the facilities for characterization.

\section{References}

[1] Ali, M., Moazzen, M., Borghei, S.M. and Taleshi, F. (2013) Change in the Morphology of ZnO Nanoparticles upon Changing the Reactant Concentration. Applied Nanoscience, 3, 295-302.

[2] Arora, A.K., Devi, S., Jaswal, V.S., Singh, J., Kinger, M. and Gupta, V.D. (2014) Synthesis and Characterization of ZnO Nanoparticles. Oriental Journal of Chemistry, 30, 1671-1679.

[3] Prasad, K. and Jha, A.K. (2009) ZnO Nanoparticles: Synthesis and Adsorption Study. Natural Science, 1, 129-135. http://dx.doi.org/10.4236/ns.2009.12016

[4] Wang, Z.L. (2004) Zinc Oxide Nanostructures: Growth, Properties and Applications. Journal of Physics: Condensed Matter, 16, R829-R858.

[5] Kulkarn, S.S. (2015) Optical and Structural Properties of Zinc Oxide Nanoparticles. International Journal of Advanced Research in Physical Science, 2, 14-18.

[6] Khan, Z.R., Arif, M. and Singh, A. (2012) Development and Study of the Structural and Optical Properties of Hexagonal ZnO Nanocrystals. International Nano Letters, 2, 22. http://www.inl-journal.com/content/2/1/22

[7] Bindu, P. and Thomas, S. (2014) Estimation of Lattice Strain in ZnO Nanoparticles: X-Ray Peak Profile Analysis. Journal of Theoretical and Applied Physics, 8, 123-134.

[8] Chen, K.J., Fang, T.H., et al. (2008) The Crystallization and Physical Properties of Al-Doped ZnO Nanoparticles. Applied Surface Science, 254, 5791-5795.

[9] Ghosh, A., Kumari, N. and Bhattacharjee, A. (2014) Investigations on Structural and Optical Properties of Cu Doped ZnO. Journal of Nano Science and Nano Technology: Spring Edition, 2, 485-489.

[10] Mukhtar, M., Munisa, L. and Saleh, R. (2012) Co-Precipitation Synthesis and Characterization of Nanocrystalline Zinc Oxide Particles Doped with $\mathrm{Cu}^{2+}$ Ions. Materials Sciences and Applications, 3, 543-551.

[11] Djaja, N.F., Montja, D.A. and Saleh, R. (2013) The Effect of Co Incorporation into ZnO Nanoparticles. Advances in Materials Physics and Chemistry, 3, 33-41.

[12] Kshirsagar, S.D., Inamdar, D., Gopalakrishnan, I.K., Kulshreshtha, S.K. and Mahamuni, S. (2007) Formation of RoomTemperature Ferromagnetic Zn1-XCoXO Nanocrystals. Solid State Communications, 143, 457-460. http://dx.doi.org/10.1016/j.ssc.2007.06.025

[13] Nair, M.G. (2011) Structural, Optical, Photo Catalytic and Antibacterial Activity of ZnO and Co-Doped ZnO Nanoparticles. Materials Letters, 65, 1797-1800.

[14] Iqbal, J., Janjua, R.A. and Jan, T. (2014) Structural, Optical and Magnetic Properties of Co-Doped ZnO Nanoparticles Prepared via Wet Chemical Route. International Journal of Modern Physics B, 28, Article ID: 1450158.

[15] Hays, J., Reddy, K.M., Graces, N.Y., Engelhard, M.H., Shutthanandan, V., Luo, M., Xu, C., Giles, N.C., Wang, C., Thevuthasan, S. and Punnoose, A. (2007) Effect of Co-Doping on the Structural, Optical and Magnetic Properties of ZnO Nanoparticles. Journal of Physics: Condensed Matter, 19, Article ID: 266203. http://dx.doi.org/10.1088/0953-8984/19/26/266203

[16] Udayakumarl, S., Renuka, V. and Kavitha, K. (2012) Structural, Optical and Thermal Studies of Cobalt Doped Hexagonal $\mathrm{ZnO}$ by Simple Chemical Precipitation Method. Journal of Chemical and Pharmaceutical Research, 4, 12711280 .

[17] Sugaharal, H.J., de Melo, E.F., de Melo, C.P. and Alvesl, K.G.B. (1994) Influence of Cobalt Doping on the Luminescence and Magnetic Properties of ZnO Nanoparticles Prepared by Co-Precipitation.

[18] Rath, C. (2009) Effect of Cobalt Substitution on Microstructure and Magnetic Properties in ZnO Nanoparticles. Indian Journal of Physics, 83, 415-421. 